\title{
Age and height dependence of lung clearance index and functional residual capacity
}

\author{
Sooky Lum*, Janet Stocks*, Sanja Stanojevic*,*, Angie Wade ", Paul Robinson*,+, \\ Per Gustafsson ${ }^{\S}$, Meghan Brown", Paul Aurora*, ${ }^{\star}$, Padmaja Subbarao\#, \\ Ah-fong $\mathrm{Hoo}^{f}$ and Samatha Sonnappa*,f
}

ABSTRACT: The lung clearance index $(\mathrm{LCl})$ is more sensitive than spirometry in detecting abnormal lung function in children with cystic fibrosis. $\mathrm{LCl}$ is thought to be independent of age, but recent evidence suggests that the upper limit of normal is higher in infants and preschool children than in older subjects. This study examines whether $\mathrm{LCl}$ remains independent of body size throughout childhood.

Multiple-breath washout data from healthy children and adolescents were collated from three centres using the mass spectrometer system and the inert gas sulfur hexafluoride. Reference equations for $\mathrm{LCl}$ and functional residual capacity (FRC) were constructed using the LMS (lambda-mu-sigma) method.

Data were available from 497 subjects (2 weeks to 19 years of age) tested on 659 occasions. $\mathrm{LCl}$ was dependent on body size, decreasing in a nonlinear pattern as height increased. Changes were particularly marked in the first 5 years of life. Height, age and sex were all independent predictors of FRC. Minimal between-centre differences allowed unified reference equations to be developed.

$\mathrm{LCl}$ is not independent of body size. Although a constant upper normal limit would suffice for cross-sectional clinical assessments from 6 years of age, appropriate reference equations are essential for accurate interpretation of results during early childhood.

KEYWORDS: Children, functional residual capacity, lung clearance index, reference values, ventilation inhomogeneity

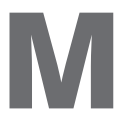
easuring the efficiency of ventilation distribution within the lung offers the exciting potential to detect early disease processes missed by conventional flow-based lung function techniques such as spirometry. Multiple-breath inert-gas washout (MBW) is a noninvasive tidal breathing test, feasible across all ages, which offers improved sensitivity, compared with spirometry, to detect early cystic fibrosis lung disease throughout childhood [1-3].

It is generally thought that LCI is independent of body size. However, cross-sectional studies in infants have suggested that the upper limit of normal (ULN) for global measures of ventilation distribution inhomogeneity, such as lung clearance index (LCI), may be higher compared with older subjects $[3,4]$. Such differences, which could arise from developmental changes or differences in measurement conditions, may preclude identification of early lung disease in both cross-sectional and longitudinal studies. Furthermore, appropriate normative data for functional residual capacity (FRC) derived from MBW (FRCMBW) spanning the paediatric age range are lacking. The aim of this study was to investigate the relationship between body size and LCI from infancy to young adulthood, and establish reference equations for both LCI and FRCMBW across this age range.

\section{METHODS}

MBW was performed in healthy children at three specialised paediatric centres in the UK, Sweden and Canada as described previously [3, 5-10]. Briefly, during wash-in, a dry air mixture containing
AFFILIATIONS

*Portex Respiratory Unit, UCL Institute of Child Health, London, "Centre for Paediatric Epidemiology and Biostatistics, UCL Institute of Child Health, London,

${ }^{f}$ Dept of Paediatric Respiratory Medicine, Great Ormond Street Hospital for Children NHS Foundation Trust, London, UK,

"Hospital for Sick Children, Toronto, Canada,

${ }^{+}$Dept of Paediatric Respiratory Medicine, The Children's Hospital, Westmead, Sydney, Australia, and ${ }^{\S}$ Dept of Paediatrics, Central Hospital, Skövde, Sweden.

\section{CORRESPONDENCE}

S. Lum

Portex Respiratory Unit

UCL Institute of Child Health

30 Guilford Street

London WC1N 1EH

UK

E-mail: s.lum@ich.ucl.ac.uk

Received:

Jan 102012

Accepted after revision:

Sept 042012

First published online:

Nov 082012 
$4 \%$ sulfur hexafluoride $\left(\mathrm{SF}_{6}\right)$ was inspired until inspiratory and expiratory $\mathrm{SF}_{6}$ concentrations were stable for a minimum of five breaths. During washout, room air was inhaled until the endtidal $\mathrm{SF}_{6}$ concentration was consistently $<0.1 \%$ (i.e. $1 / 40$ of the starting concentration). Mean FRC and LCI were reported from three technically acceptable runs. Data were collected between 2000 and 2010 using a respiratory mass spectrometer (AMIS 2000; Innovision A/S, Odense, Denmark) with identical MBW system design and analysis software (developed by $\mathrm{P}$. Gustafsson) and protocols in all centres [11]. Please see the online supplement for details of equipment and software indicators. The inclusion criteria for healthy children were the same across all three sites $[2,3,7,8,10,12]$. Subjects were free from respiratory illness for $\geqslant 3$ weeks. Infants and young children $<2$ years of age were studied supine and in quiet sleep following light sedation with chloral hydrate using a facemask. Preschool (3-6 years) and school age ( $>6$ years) children were tested sitting upright and awake using a facemask and mouthpiece, respectively. Data from London, UK and Sweden were collected using a Fleisch pneumotachometer (PNT) while a Hans Rudolph PNT (Hans Rudolph Inc., Shawnee, KS, USA) was used in Toronto, Canada (see online supplementary material for details of methodological differences between centres). In London and Toronto, healthy children were followed longitudinally as part of observational studies. The timing of repeated measurements varied according to study protocol, and ranged from 2 weeks to 6 years. All research studies were approved by the local research ethics committees of participating hospitals, and informed written consent obtained from all parents and assent from older children.

\section{Statistical analysis}

Population characteristics were summarised using descriptive statistics. Centre differences were explored using ANOVA or Chi-squared analysis where appropriate. The relationship between body size and MBW outcomes was initially explored by visual inspection of data. Reference equations for both LCI and FRCMBW were constructed as described previously [13-15] using the LMS (lambda-mu-sigma) method [16]. This method is an extension of regression analysis that includes three components: 1) skewness (lambda, $L$ ), which models the departure of variables from normality using a Box-Cox transformation; 2) median $(\mathrm{mu}, \mathrm{M})$ or predicted value; and 3) coefficient of variation (sigma, $S$ ), which models the spread of values around the median and adjusts for any nonuniform dispersion. The three quantities are allowed to change with height and/or age, to reflect changes in the distribution as children grow. The $L, M$ and $S$ coefficients are combined algebraically to convert individual observations to z-scores [16]:

$$
\begin{aligned}
\text { z-score } & =\left((\text { measurement } / M)^{L}-1\right) / L \times S \\
\mathrm{ULN} & =M \times(1.96 \times S \times L+1)^{1 / L}
\end{aligned}
$$

We applied the LMS method using the GAMLSS package [17] in $\mathrm{R}$ (version 2.6.1; R Foundation, Vienna, Austria). In these analyses, we also used the random function to adjust for repeated measurements in individuals. Fractional polynomials [18], whereby a combination of integer or fractional power terms are fitted to produce a polynomial equation, were used to fit each curve to explain body size-related changes. Goodness of fit was assessed using the Schwarz Bayesian criterion, which compares consecutive models directly while adjusting for increased complexity to determine the simplest model with best fit [19].

Although conventionally, the ULN is a z-score of 1.64, as this was an epidemiological study, where the cost and consequences of false-positive and false-negative test results are over-riding, an ULN corresponding to the 97.5th centile (z-score 1.96) was used as recommended by the Global Lung Function Initiative [20].

Data storage and management at the UCL Institute for Child Health (ICH), London were undertaken using Re-Base ${ }^{\mathrm{TM}}$ software (Re-Base Ltd, London, UK).

\section{RESULTS}

\section{Population characteristics}

LCI data were available from 497 subjects on 659 test occasions from 2 weeks to 19 years of age (table 1 ). There were 201 observations from infants and children $<2$ years of age $(49 \%$ males), 138 between 3 and 6 years of age (44\% males), and 320 from those $>6$ years of age ( $47 \%$ males). Although the majority of subjects were measured on a single test occasion, $22 \%$ had two observations, $11 \%$ had three or four observations, while eight $(2 \%)$ children had five or six observations. When compared against subjects from London or Toronto, Swedish subjects were older $(\mathrm{p}<0.0001$ for both centres) and, after adjusting for sex and age [21], significantly heavier $(p=0.002$ and $\mathrm{p}=0.03$, respectively).

\section{Lung clearance index}

LCI decreased nonlinearly with increasing age and height, with no significant association with sex. In a multivariable regression model, after adjusting for height, age was no longer an independent predictor of LCI. The simplest model that explained the greatest variability of LCI was used to define the reference equation for LCI and, hence, in the final model, only height was included (table 2). The relationship between height and LCI is presented in figure 1, as the fitted reference equation (50th centile) together with the ULN (97.5th centile) and lower limit of normal (2.5th centile). Table 3 shows examples of predicted LCI and the ULN for LCI according to height. After correction for height, no significant intersubject differences were found between LCI measurements made supine (i.e. sedated infants) and older subjects who were studied sitting (adjusted $\beta=0.14,95 \%$ CI $-0.07-0.34$; fig. S1), or between those obtained with a mask (infants and preschool children) or mouthpiece (older children) (adjusted $\beta=-0.11$, $95 \%$ CI -0.23-0.01). After the best model was determined, centre differences were tested as fixed effects within the model. There were small, albeit statistically significant, differences between centres, with Toronto having slightly lower LCI results (mean difference $-0.35,95 \%$ CI $-0.45--0.24$ ) than either London or Sweden. Sensitivity analysis found the same nonlinear relationship with height and minimal effect $(<1 \%)$ on reported ULN if Toronto data were excluded.

The majority $(80 \%)$ of children were of white European origin, the remainder being black African or Caribbean (5.6\%), Asian $(7.3 \%)$ or of a variety of other ethnic origins $(7.1 \%)$. When the sample was dichotomised into "white" and "non-white", no statistical differences in LCI were observed between groups 
TABLE 1 Population characteristics

\begin{tabular}{lcccc} 
Background details & London, UK & Skövde, Sweden & Toronto, Canada & Total population \\
\hline Subjects $\mathbf{n}($ males \%) & $293(47)$ & $102(45)$ & $102(52)$ & $46(75)$ \\
White $\mathbf{n}(\%)$ & $220(75)$ & $102(100)$ & 114 & $397(48)$ \\
Test occasions $\mathbf{n}$ & 443 & 102 & $1.6(0.23-18.9)$ & 559 \\
Age years & $5.2(0.05-16.0)$ & $17.9(4.5-18.7)$ & $11.1(5.2-98.7)$ & $20.7(3.7-101.0)$ \\
Weight kg & $19.1(3.7-71.9)$ & $63.0(20.5-101.0)$ & $0.24 \pm 1.14$ & $0.28 \pm 1.02$ \\
Weight z-score & $0.22 \pm 1.00$ & $0.59 \pm 0.93$ & $82.8(60.0-185.5)$ & $114.7(52.5-196.5)$ \\
Height cm & $110.5(52.5-183.0)$ & $169.3(113.2-196.5)$ & $0.32 \pm 1.02$ & $0.41 \pm 1.03$ \\
Height z-score & $0.39 \pm 1.04$ & $0.59 \pm 0.93$ & & \\
\hline
\end{tabular}

Data presented as median (range) or mean $\pm \mathrm{SD}$, unless otherwise stated. * : adjusted according to British 1990 growth charts [21].

(mean difference 0.05, 95\% CI -0.17-0.07). The sample size for different ethnic groups was too small to investigate changes in LCI in relation to height within each group separately $(<40$ in three groups and $<10$ in the remaining three).

\section{$\mathbf{L C l}$ in children $>6$ years of age}

As can be seen from figure 1, despite a continuing small reduction in LCI throughout the entire paediatric range, changes were minimal once the child attained a height of $\sim 115 \mathrm{~cm}$ ( $\sim 6$ years of age). LCI data from children $>6$ years of age were, therefore, examined separately. These were available from 255 subjects on 316 test occasions of which $55(17 \%)$ datasets were collected using a facemask. Within this subgroup, there was no significant association between LCI and either height or age, or whether a mask or mouthpiece was used. Mean \pm SD LCI for children 6-19 years of age was $6.54 \pm 0.51$, with an ULN $(\mathrm{ULN}=$ mean $+1.96 \times \mathrm{SD})$ for this age group of

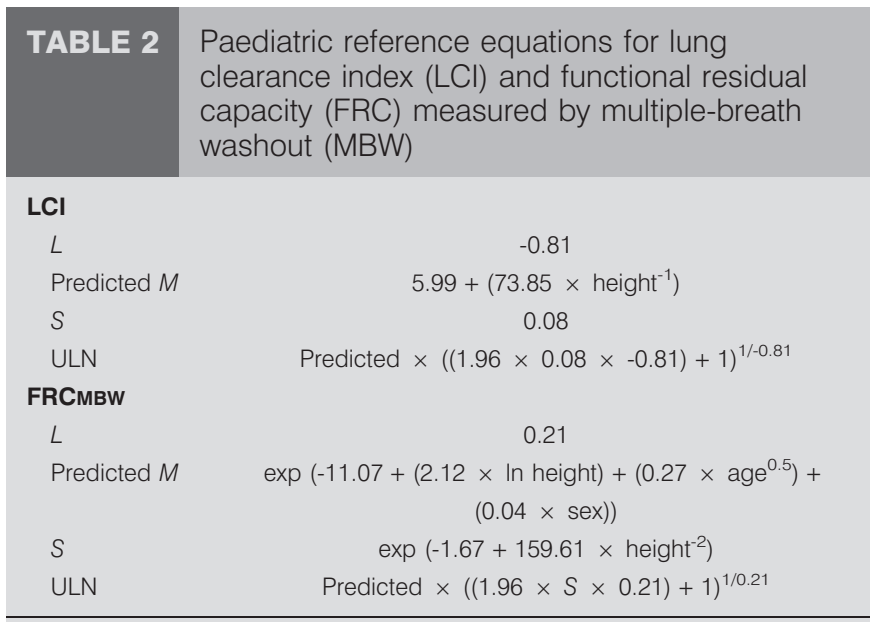

Based on these equations, the upper limit of normal (ULN) for LCl can be simplified to predicted $\mathrm{LCI} \times 1.18$, whereas that for FRCMBW becomes $(0.36 \times S+1)^{5.37} \times$ predicted FRCMBW. For simplicity, the factors are displayed to two decimal places. For calculation of z-scores and the ULN, the full equations presented in table S3 should be used. L: lambda (skewness); $M:$ mu (mean); S: sigma (coefficient of variation); height: height in $\mathrm{cm}$; age: age in years; sex: female $=0$, male $=1$.
7.56 if a fixed threshold were to be used (fig. 2). A similar pattern was seen plotting LCI against height (data not shown).

\section{FRC measured by MBW}

FRCMBW data were available from 469 subjects on 631 test occasions, as FRC had not been originally recorded separately for 28 Swedish subjects and could not be retrieved subsequently due to software and equipment upgrades. Since FRCMBW was heavily right skewed, log-transformed FRCMBW values were used in the models. Using multivariable models, height, age and sex were all independent predictors of FRCMBW. In addition, variability around the mean varied with height and there was residual skewness, both of which were adjusted for in the models. The relationship between FRCMBW and height is presented in figure 3. There was, on average, a 30-fold increase in FRCMBW during the first 19 years of life. After adjustment for height, age and sex, children from Sweden and Toronto had slightly higher values of FRCMBW compared with London: mean difference (95\% CI) 0.05 (-0.02-0.12) and $0.05(0.01-0.09) \mathrm{L}$, respectively. No significant differences in FRCMBW were detected between non-white children and white children (mean difference $0.01(-0.02-0.05) \mathrm{L})$. By contrast, the type of equipment and posture were both significant in the FRCMBW model, mean values being $0.12(0.07-0.17)$ L higher when a mask rather than mouthpiece was used and $0.14(0.08-0.21)$ L lower in the supine than the sitting posture, after adjusting for height, age and sex.

Predicted FRCMBW from the current study was fairly similar to that derived from helium dilution equations [22], despite the use of different techniques and a different inert gas (table S2). Table 4 shows examples of predicted values and ULN for FRCMBW according to height and sex.

\section{DISCUSSION}

Contrary to the widely held belief that LCI remains constant in health, significant reductions in LCI occur with growth, which are particularly marked in the first 5 years of life. Use of a fixed ULN creates two sources of error with clinical implications: overdiagnosis of abnormal ventilation distribution in healthy infants and young children; and poor sensitivity to detect worsening lung function (relative to the true change in ULN) when absolute values either remain stationary over time or decline by less than the predicted amount. It is well recognised that relative independence of LCI from body size, due to the 


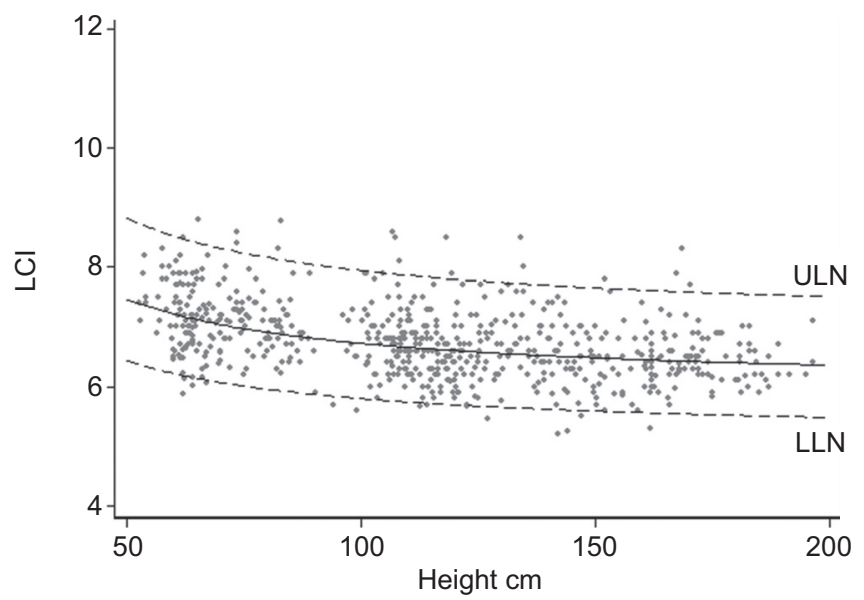

FIGURE 1. Lung clearance index (LCI) from infancy to 19 years of age. The solid line denotes the predicted (50th centile) LCI for height and the dashed lines denote the upper limit of normal (ULN; 97.5th centile) and lower limit of normal (LLN; 2.5th centile)

intrinsic correction for FRC, is of considerable advantage when interpreting results, particularly in children with respiratory disease who may have accompanying impairments of somatic growth [1, 23]. Magnitude of change in LCI over the first 19 years of life is minimal when compared with the 30-fold increase observed for lung volumes and airflows over this period [24]. Nevertheless, observed changes in LCI during early childhood, with an absolute decrease in ULN by $>0.8$ between 1 month and 5 years of age $(\sim 110 \mathrm{~cm})$, are large enough to be of clinical significance and could bias attempts to track lung function between infancy, the preschool years and later childhood.

Despite frequent reference to the stability of LCI in health across age ranges, close inspection of the literature reveals a range of values according to the age of the population studied, with the ULN ranging from 7.8 (using mass spectrometry and $\mathrm{SF}_{6}$ ) to 8.2 (using an ultrasonic flowmeter and $\mathrm{SF}_{6}$ ) during infancy $[1,3,25,26], 7.4$ to 7.8 in preschoolers $[1,27]$ and 7.2 to 7.4 in older school children $[2,5]$, which is consistent with the significant inverse association of LCI with height across the paediatric age range found in this study. Nevertheless, beyond 6 years of age $(\sim 115 \mathrm{~cm}$ in height), LCI did stabilise with $<0.3$ further reduction in the ULN until early adulthood. Indeed, when analysis was restricted to children $>6$ years of age, no significant relationship with height or age was observed.

The use of a fixed LCI ULN of 7.53 in older children (fig. 2) would increase the risk of overdiagnosis of abnormality in children $<10$ years of age (i.e. below $\sim 140 \mathrm{~cm}$ ), with differences of up to 0.3 between the fixed and "true" ULN derived from all subjects (table 3). However, given the marked elevations in LCI reported in cystic fibrosis once school age is reached [2, 5, 28, 29], this is likely to have minimal clinical impact in cross-sectional measurements. By contrast, when interpreting longitudinal changes within the same child at any age, the use of LCI expressed as zscores is advised, as the ULN changes with body size.

Although predicted LCI and FRCMBW values are likely to be both device- and inert gas-specific [23], the changes in LCI that

\begin{tabular}{|c|c|c|}
\hline TABLE 3 & \multicolumn{2}{|c|}{$\begin{array}{l}\text { Examples of predicted lung clearance index } \\
\text { (LCl) and the upper limit of normal (ULN) of LCl } \\
\text { according to height }\end{array}$} \\
\hline Height $\mathrm{cm}$ & Predicted LCI & ULN of LCI \\
\hline 55 & 7.33 & 8.67 \\
\hline 60 & 7.22 & 8.54 \\
\hline 65 & 7.13 & 8.43 \\
\hline 70 & 7.04 & 8.33 \\
\hline 80 & 6.91 & 8.17 \\
\hline 100 & 6.73 & 7.96 \\
\hline 120 & 6.60 & 7.81 \\
\hline 140 & 6.52 & 7.71 \\
\hline 160 & 6.45 & 7.63 \\
\hline \multicolumn{3}{|c|}{$\begin{array}{l}\text { As a broad approximation, } 55 \mathrm{~cm} \text { represents average height (50th centile) at } \\
5 \text { weeks, } 60 \mathrm{~cm} \text { at } 3 \text { months, } 70 \mathrm{~cm} \text { at } 9 \text { months, } 80 \mathrm{~cm} \text { at } 18 \text { months, } 100 \mathrm{~cm} \\
\text { at } 4 \text { years, } 120 \mathrm{~cm} \text { at } 7 \text { years and } 140 \mathrm{~cm} \text { at } 10 \text { years. After the age of } 10 \text { years, } \\
\text { height becomes highly dependent on sex as well as age, the average height of } \\
\text { an } 18 \text {-year-old male and female being } 177 \text { and } 164 \mathrm{~cm} \text {, respectively. }\end{array}$} \\
\hline
\end{tabular}

we have observed during early childhood are likely to be relevant irrespective of the particular technique used. When using ultrasonic flow meter technology in infants, an ULN $>8$ has been reported [26, 30]. Furthermore, CHAKR et al. [31] recently demonstrated that gas mixing within the lung becomes more homogeneous with increasing age early in life, using the LCI and phase III slopes. This is consistent with our current findings based on mass spectrometry. Likewise, the ULN for LCI reported for subjects $>5$ years of age when using a photoacoustic $\mathrm{SF}_{6}$ gas analyser and Hans Rudolph PNT [29] or ultrasonic flow meter technology $[28,32]$ is similar to that reported here. While FUCHS and co-workers [28, 32] found LCI

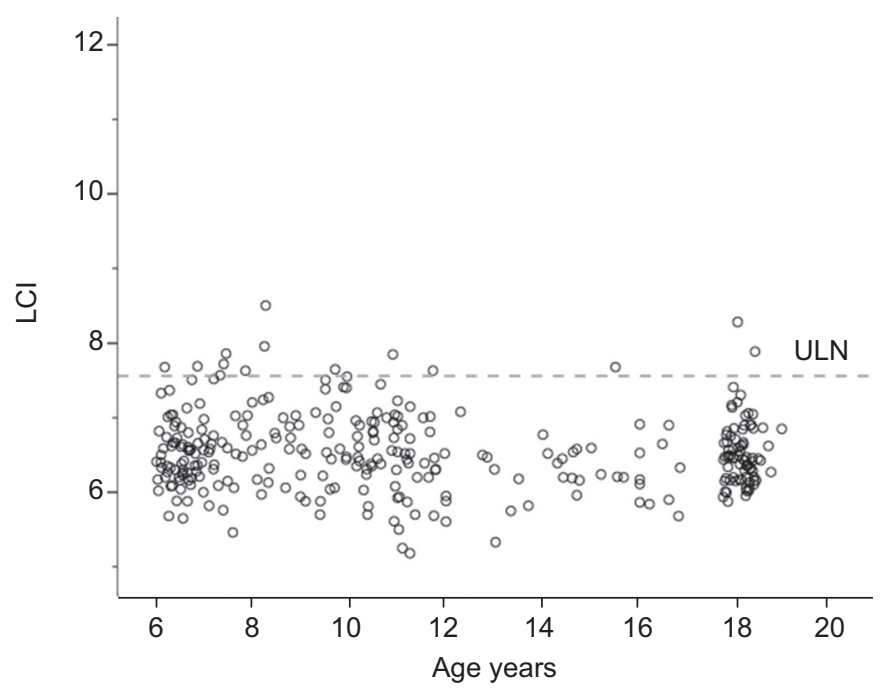

FIGURE 2. Lung clearance index (LCl) plotted against age in subjects $>6$ years of age. When the analysis was limited to children $>6$ years, $\mathrm{LCl}$ was independent of both age and height, such that a constant upper limit of normal (ULN) of 7.56 could be used for cross-sectional assessments between 6 and 19 years of age. 


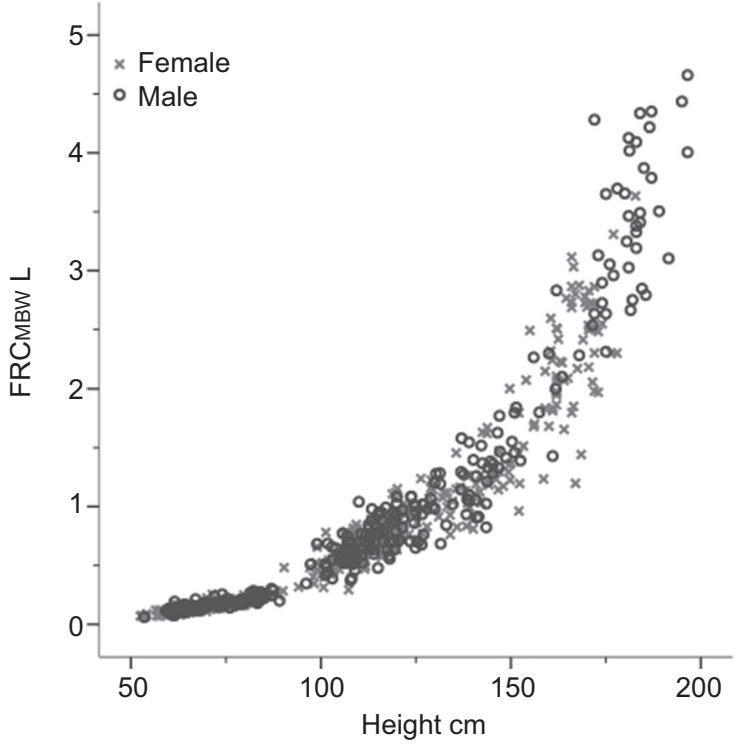

FIGURE 3. Relationship between functional residual capacity (FRC) measured by multiple-breath washout (MBW) and body size.

to be independent of growth between 5 and 18 years, LCI was found to increase slightly in healthy subjects between 6 and 58 years of age [29], a pattern also observed by ROBINSON et al. [23] when comparing published data across a wide age range. Recently, age dependency of LCI during adulthood (between 25 and 65 years of age) has been reported, showing that gas mixing becomes more heterogeneous with age [33]. This suggests that gas mixing is more heterogeneous both in the very young and in elderly adults, although formal longitudinal studies across wide age ranges are required to confirm this.

Measurement conditions did influence FRCMBW, with higher FRCMBW values in facemask tests (despite attempts to minimise apparatus deadspace and correction for such deadspace) and in the sitting position. These influences are inevitable when transitioning from early to late childhood and must be reflected in the prediction equations used. The magnitude of the effect is unclear in early childhood and a number of factors may act in different directions. Increased equipment deadspace may cause compensatory increase in tidal volume, and dynamic elevation of end-expiratory level, especially during infancy [34]. Minimisation of deadspace is recommended because in young children, increased deadspace and hypercapnia leads to deeper tidal volume and increased tendency toward dynamic hyperinflation of FRC. This may explain the intercentre differences (ICH versus Toronto) in LCI and FRCMBW results observed during infancy [35]. Equipment deadspace is larger with a facemask as opposed to a mouthpiece and increased anatomical deadspace in relation to lung volume, especially in infants and young children, could adversely affect gas mixing efficiency. However, the magnitude of the effect has been investigated recently in infants, by using alveolar-based indices corrected for deadspace, and did not account for the increased LCI pattern seen in early life [36].

In the current study, choice of interface did not have a significant effect on children $>6$ years of age, where numbers were large enough to allow comparison. Minimal atelectasis in the dependent lung regions of supine infants and the effects of gravity may, in theory, increase LCI, but would not explain the growth-related changes observed during infancy. We speculate that these growth-related changes, in part, reflect the rapid alveolarisation that occurs during infancy and early childhood $[3$, 37], with more heterogeneous gas distribution due to asymmetrical branching within the acinar regions in younger subjects.

A small study of six healthy adults using nitrogen washout suggested that LCI increased significantly in the supine and head-down postures, supporting the view that gas distribution is less uniform in such postures than when upright [38, 39]. However, preliminary data from cystic fibrosis subjects, possibly due to the reduced FRC and/or due to previously less ventilated apical lung regions becoming more ventilated,

TABLE 4 Examples of predicted functional residual capacity (FRC) measured by multiple-breath washout (MBW) and the upper limit of normal (ULN) for FRCMBW according to height and sex

\begin{tabular}{|c|c|c|c|c|}
\hline \multirow[t]{2}{*}{ Height $\mathrm{cm}$} & \multicolumn{2}{|c|}{ Males } & \multicolumn{2}{|c|}{ Females } \\
\hline & Predicted FRCмвш L & ULN for FRCмвш L & Predicted FRCмвW L & ULN for FRCмвш L \\
\hline 55 & 0.085 & 0.123 & 0.082 & 0.119 \\
\hline 60 & 0.107 & 0.154 & 0.103 & 0.149 \\
\hline 65 & 0.132 & 0.189 & 0.128 & 0.184 \\
\hline 70 & 0.162 & 0.232 & 0.158 & 0.226 \\
\hline 80 & 0.237 & 0.338 & 0.229 & 0.327 \\
\hline 100 & 0.463 & 0.660 & 0.447 & 0.636 \\
\hline 110 & 0.622 & 0.885 & 0.598 & 0.851 \\
\hline 120 & 0.816 & 1.161 & 0.784 & 1.115 \\
\hline 140 & 1.339 & 1.903 & 1.276 & 1.814 \\
\hline 160 & 2.024 & 2.874 & 1.966 & 2.792 \\
\hline
\end{tabular}

To take age into account, results have been calculated by using the age at which the quoted values for height represent the 50 th centile. Thus, $70 \mathrm{~cm}$ represents the 50 th centile for height at 0.64 years in males but 0.75 years in females, whereas $160 \mathrm{~cm}$ represents the 50 th centile for height at 13.7 years in boys but 14.1 years in females [21]. 
this effect is not seen in young sedated infants and toddlers with cystic fibrosis [40]. Imaging studies in children demonstrate atelectasis in dependent lung regions when supine [41, 42]. The effect of sedation on ventilation distribution inhomogeneity remains unclear, although values in healthy unsedated infants $[26,30]$ are similar to the results reported here in sedated infants, despite different systems. Chloral hydrate sedation has been shown to have minimal effects on breathing patterns, strength of Hering-Breuer inflation reflex or oxygen saturation in healthy infants during the first 2 years of age [43, 44].

Irrespective of the exact contributions of these underlying mechanisms, it is evident that LCI values are elevated during early childhood and that prediction equations reflecting the changes occurring with growth are required. The reference equations presented in this paper are unique and, for the first time, contain sufficient numbers across a wide enough age range to provide smoothly changing curves describing the transition between infancy, preschool and school-age children without arbitrary break points at different ages or body size, thereby eliminating the need to switch between equations $[45,46]$. This was achieved by collating data from three centres using almost identical equipment. The lower LCI observed in Toronto may have been due to the relatively large equipment deadspace (table S1) resulting in higher FRCMBW when compared with the ICH data. Despite small intercentre differences, the ability to combine these data to form unified equations suggests that they can be generalised to other populations using similar equipment and analysis protocols. The height dependency of LCI observed in this dataset is likely to be relevant irrespective of the techniques used but the extent to which these equations are applicable to newer commercially available equipment [32], especially those based on nitrogen washout [47-49], will need to be determined.

In conclusion, LCI is not constant throughout childhood and, while the use of fixed ULNs for assessments on single occasions in children $>6$ years of age may not have any significant clinical impact on interpretation, this is not appropriate for younger children or those in whom serial measurements are being undertaken at any age. Expression of results as z-scores, using prediction equations reflecting the developmental changes occurring across childhood, will allow more accurate interpretation of LCI results in children.

\section{SUPPORT STATEMENT}

This study was supported by the UK Medical Research Council (grant G0401525), the Cystic Fibrosis Trust (PJ550), a Wellcome Trust Value in People award (to S. Lum), the British Lung Foundation (P06/7 and P99/16), Asthma UK (06/037 and 10/013) and Smiths Medical PLC; research at the Institute of Child Health and Great Ormond Street Hospital for Children NHS Trust (London, UK) benefits from research and development funding from the NHS Executive, the Canadian Institute of Health Research (AEC-85761) and AllerGEN NCE (09A7).

\section{STATEMENT OF INTEREST}

None declared.

\section{ACKNOWLEDGEMENTS}

The authors thank all the children and families who kindly participated in our epidemiological studies, K. Costeloe (Homerton University NHS Trust, London, UK), H. Ljungberg, D. Ahmed, J. Chudleigh, C. Oliver, C. Saunders, W. Kozlowska, J. Kirkby, L. Walsh, C. Bastardo (all Portex
Respiratory Unit, Institute of Child Health, London) and the CHILD Toronto Study team for recruitment and data collection.

\section{REFERENCES}

1 Aurora P, Kozlowska W, Stocks J. Gas mixing efficiency from birth to adulthood measured by multiple-breath washout. Respir Physiol Neurobiol 2005; 148: 125-139.

2 Gustafsson PM, Aurora P, Lindblad A. Evaluation of ventilation maldistribution as an early indicator of lung disease in children with cystic fibrosis. Eur Respir J 2003; 22: 972-979.

3 Lum S, Gustafsson P, Ljungberg H, et al. Early detection of cystic fibrosis lung disease: multiple-breath washout versus raised volume tests. Thorax 2007; 62: 341-347.

4 Sinhal S, Galati J, Baldwin DN, et al. Reproducibility of multiple breath washout indices in the unsedated preterm neonate. Pediatr Pulmonol 2010; 45: 62-70.

5 Aurora P, Gustafsson P, Bush A, et al. Multiple breath inert gas washout as a measure of ventilation distribution in children with cystic fibrosis. Thorax 2004; 59: 1068-1073.

6 Brown M, Balkovec S, Keast C, et al. Lung clearance index as a marker of peripheral airways disease in children under 5 years of age. Am J Respir Crit Care Med 2010; 181: A5968.

7 Brown M, Jensen R, Balkovec S, et al. Effects of forced expiratory maneuvers and bronchodilator on lung clearance index in infants. Am J Respir Crit Care Med 2011; 183: A6311.

8 Sigurs N, Aljassim F, Kjellman B, et al. Asthma and allergy patterns over 18 years after severe RSV bronchiolitis in the first year of life. Thorax 2010; 65: 1045-1052.

9 Sonnappa S, Bastardo CM, Wade A, et al. Symptom-pattern phenotype and pulmonary function in preschool wheezers. J Allergy Clin Immunol 2010; 126: 519-526.

10 Aurora P, Stanojevic S, Wade A, et al. Lung clearance index at 4 years predicts subsequent lung function in children with cystic fibrosis. Am J Respir Crit Care Med 2011; 183: 752-758.

11 Aurora P, Bush A, Gustafsson P, et al. Multiple-breath washout as a marker of lung disease in preschool children with cystic fibrosis. Am J Respir Crit Care Med 2005; 171: 249-256.

12 Lum S, Kirkby J, Welsh L, et al. Nature and severity of lung function abnormalities in extremely pre-term children at 11 years of age. Eur Respir J 2011; 37: 1199-1207.

13 Cole TJ, Stanojevic S, Stocks J, et al. Age- and size-related reference ranges: a case study of spirometry through childhood and adulthood. Stat Med 2009; 28: 880-898.

14 Stanojevic S, Wade A, Stocks J, et al. Reference ranges for spirometry across all ages: a new approach. Am J Respir Crit Care Med 2008; 177: 253-260.

15 Stanojevic S, Wade A, Cole TJ, et al. Spirometry centile charts for young Caucasian children: the Asthma UK Collaborative Initiative. Am J Respir Crit Care Med 2009; 180: 547-552.

16 Cole TJ, Green PJ. Smoothing reference centile curves: the LMS method and penalized likelihood. Stat Med 1992; 11: 1305-1319.

17 Rigby RA, Stasinopoulos DM. Smooth centile curves for skew and kurtotic data modelled using the Box-Cox power exponential distribution. Stat Med 2004; 23: 3053-3076.

18 Royston P, Altman DG. Regression using fractional polynomials of continuous covariates: parsimonious parametric modelling. Appl Stat 1994; 43: 429-467.

19 Schwarz G. Estimating the dimension of a model. Ann Stat 1978; 6: 461-464.

20 Quanjer PH, Stanojevic S, Cole TJ, et al. Multi-ethnic reference values for spirometry for the 3-95 year age range: the global lung function 2012 equations. Eur Respir J 2012; 40: 1324-1343.

21 Cole TJ, Freeman JV, Preece MA. British 1990 growth reference centiles for weight, height, body mass index and head circumference fitted by maximum penalized likelihood. Stat Med 1998; 17: 407-429. 
22 Stocks J, Quanjer PH. Reference values for residual volume, functional residual capacity and total lung capacity. Eur Respir J 1995; 8: 492-506.

23 Robinson PD, Latzin P, Gustafsson PM. Multiple-breath washout. Eur Respir Monogr 2010; 47: 87-104.

24 Lum S, Stocks J. Forced expiratory manoeuvres. Eur Respir Monogr 2010; 47: 46-65.

25 Fuchs O, Latzin $\mathrm{P}$, Thamrin $\mathrm{C}$, et al. Normative data for lung function and exhaled nitric oxide in unsedated healthy infants. Eur Respir J 2011; 37: 1208-1216.

26 Hulskamp G, Lum S, Stocks J, et al. Association of prematurity, lung disease and body size with lung volume and ventilation inhomogeneity in unsedated neonates: a multicentre study. Thorax 2009; 64: 240-245.

27 Sonnappa S, Bastardo C, Stafler P, et al. Ethnic differences in FeNO and lung function in healthy young children. Chest 2011; 140: 1325-1331.

28 Fuchs SI, Sturz J, Junge S, et al. A novel sidestream ultrasonic flow sensor for multiple breath washout in children. Pediatr Pulmonol 2008; 43: 731-738.

29 Horsley AR, Gustafsson PM, Macleod KA, et al. Lung clearance index is a sensitive, repeatable and practical measure of airways disease in adults with cystic fibrosis. Thorax 2008; 63: 135-140.

30 Latzin P, Roth S, Thamrin C, et al. Lung volume, breathing pattern and ventilation inhomogeneity in preterm and term infants. PLoS One 2009; 4: e4635.

31 Chakr VC, Llapur CJ, Sarria EE, et al. Ventilation homogeneity improves with growth early in life. Pediatr Pulmonol 2012; 47: 373-380.

32 Fuchs SI, Eder J, Ellemunter H, et al. Lung clearance index: Normal values, repeatability, and reproducibility in healthy children and adolescents. Pediatr Pulmonol 2009; 44: 1180-1185.

33 Verbanck S, Thompson BR, Schuermans D, et al. Ventilation heterogeneity in the acinar and conductive zones of the normal ageing lung. Thorax 2012; 67: 789-795.

34 Stocks J, Hislop AA. Structure and function of the respiratory system: developmental aspects and their relevance to aerosol therapy. In: Bisgaard H, O'Callaghan C, Smaldone GC, eds. Drug Delivery to the Lung: Clinical Aspects. New York, Marcel Dekker Inc., 2001; pp. 47-104.

35 Beydon N, Davis SD, Lombardi E, et al. An official American Thoracic Society/European Respiratory Society statement: pulmonary function testing in preschool children. Am J Respir Crit Care Med 2007; 175: 1304-1345.
36 Haidopoulou K, Lum S, Stocks J, et al. Alveolar LCI vs. standard LCI in detecting early CF lung disease. Respir Physiol Neurobiol 2012; 180: 247-251.

37 Schulzke SM, Hall GL, Nathan EA, et al. Lung volume and ventilation inhomogeneity in preterm infants at 15-18 months corrected age. J Pediatr 2010; 156: 542-549.

38 Bouhuys A, van Lennep HJ. Effect of body posture on gas distribution in the lungs. J Appl Physiol 1962; 17: 38-42.

39 Gronkvist M, Bergsten E, Gustafsson PM. Effects of body posture and tidal volume on inter- and intraregional ventilation distribution in healthy men. J Appl Physiol 2002; 92: 634-642.

40 Aljassim F, Lindblad A, Gustafsson PM. Effects of body posture on FRC and LCI in CF chilldren. Am J Respir Crit Care Med 2009; 179: A1776.

41 Lutterbey G, Wattjes MP, Doerr D, et al. Atelectasis in children undergoing either propofol infusion or positive pressure ventilation anesthesia for magnetic resonance imaging. Paediatr Anaesth 2007; 17: 121-125.

42 Sarria EE, Mattiello R, Rao L, et al. Computed tomography score and pulmonary function in infants with chronic lung disease of infancy. Eur Respir J 2011; 38: 918-923.

43 Jackson EA, Rabbette PS, Dezateux CA, et al. The effect of triclofos sodium sedation on respiratory rate, oxygen saturation and heart rate in infants and young children. Pediatr Pulmonol 1991; 10: $40-45$.

44 Rabbette PS, Dezateux CA, Fletcher ME, et al. Influence of sedation on the Hering-Breuer Inflation Reflex in healthy infants. Pediatr Pulmonol 1991; 11: 217-222.

45 Kirkby J, Aurora P, Spencer H, et al. "Stitching and switching": the impact of discontinuous lung function reference equations. Eur Respir J 2012; 39: 1256-1257.

46 Stanojevic S, Wade A, Stocks J. Reference values for lung function: past, present and future. Eur Respir J 2010; 36: 12-19.

47 Fuchs SI, Buess C, Gappa M. In vitro validation of nitrogen multiple breath washout using ultrasonic equipment. Eur Respir J 2011; 38: Suppl. 55, 355s.

48 Singer F, Abbas C, Stern G, et al. Double tracer gas single breath washout - comparison with conventional lung function tests in children with and without cystic fibrosis. Eur Respir J 2011; 38: Suppl. 55, 354s.

49 Viklund E, Lindblad A, Robinson PD, et al. Comparison of a new nitrogen multiple breath washout method to mass spectrometer $\mathrm{SF}_{6}$ washout in cystic fibrosis subjects. Eur Respir J 2011; 38: Suppl. 55,354 s. 